\title{
Contrast Enhancement of Power Plant Images Obtained from Industrial Borescope Devices
}

Reda ammar

Dept. of Electronics and Electrical Communications Engineering., Faculty of Elect., Eng., Menoufia University, Egypt.

Waleed el-shafai

Dept. of Electronics and Electrical Communications Engineering., Faculty of Elect., Eng., Menoufia University, Egypt.
Amir elsafrawey

Dept. of Electronics and Electrical Communications Engineering., Faculty of Elect., Eng., Menoufia University, Egypt.

Atef abou elazm

Dept. of Electronics and Electrical Communications Engineering., Faculty of Elect., Eng., Menoufia University, Egypt.
Huda ashiba

Dept. of Electronics and Electrical Communications Engineering., Faculty of Elect., Eng., Menoufia University, Egypt.

Fathi abd el samie

Dept. of Electronics and Electrical Communications Engineering., Faculty of Elect., Eng., Menoufia University, Egypt.

\begin{abstract}
The industrial Video scope (VS) device is designed for the endoscopic inspection of professional equipment, like motors, pumps, turbines, cavities in buildings and vehicle bodies, etc. The output of this device is images and videos, but some output images may suffer from low contrast and poor details. This paper presents three proposed approaches to enhance the quality of the VS images. The first proposed approach is based on Contrast Limited Adaptive Histogram Equalization (CLAHE) with adaptive gamma correction. By choosing the best clip limits in CLAHE and transfer function (adaptive gamma correction), the first proposal achieves optimum contrast enhancement for the VS images. The second proposed approach depends on the Homomorphic with emphasis high pass filter. The homomorphic filtering is utilized on the video scope image in logarithm domain, Applying this method gives more details in the VS images. The third proposed approach depends on Homomorphic with emphasis high pass filter with histogram equalization. Applying this method gives more accurate and best visual quality of the output VS images. Numerical results show the ability of the three proposals in improvement the VS image quality, and they can be recommended for industrial VS systems.
\end{abstract}

Keywords Videoscope images, CLAHE, Homomorphic filtering, adaptive gamma correction, Histogram equalization.

\section{Introduction}

Industrial videoscope devices (borescope) are designed for endoscopic inspection of technical units such as turbines, engines, motors, pumps, cavities in buildings and vehicle bodies, etc. [1].
The contrast enhancement is an important process for both videos and images, many methods of improving contrast were studied to improve visual image quality. And classification of these techniques into two major groups; the first group is the spatial domain and the second is the transformation or frequency domain group. [2].

The first, spatial domain group includes the Histogram Equalization (HE) methods, which can be further, categorized into two categories:

1. Global HE (GHE): it finds out the information of histogram for the entire image to configure its transformation function [3].

- Histogram Modification (HM) techniques are a part of the GHE technology.

- Gamma correction methods are a type of (HM) that is flexibly obtained using a variable gamma correction [4].

2. Local HE (LHE): it can enhance the local details of image effectively. LHE optimization methods generally require more calculations than GHE methods.

The second, transformation or frequency domain the image is first analyzed before enhancement to reduce artifacts and improve visual quality such as Homomorphic filtering [5].

This paper presents three proposed approaches enhancement methods.

- The first proposed approach named CLAHE with adaptive gamma correction. By specifying the best clip limit in CLAHE and transfer function adaptive gamma correction the first proposed method achieves optimum contrast enhancement for the VS images.

- The second proposed approach depends on the Homomorphic with emphasis high pass filter. The homomorphic filtering is performed on the VS image in the logarithm domain by analyzing the image into two component illumination component and 
reflectance components. Applying this method gives more details in the VS image.

- The third proposed approach depends on Homomorphic with emphasis high pass filter and histogram equalization. The emphasis high pass filter is executed for the videoscope image in logarithm domain, and Apply histogram equalization on the output image, we get high quality and more details for the VS image.

This paper is organized as follows. Section 2 shows the relevant works. Section 3 shows the proposed approaches. Section 4 the performance evaluation survey. Section 5 shows concluding observations.

\section{Relevant works}

A. This section presents a simplified review of previous studies to enhance images that are related to CLAHE with adaptive gamma correction that are histogram equalization, histogram matching, gamma correction, and CLAHE.

\subsection{Histogram equalization}

The histogram shows as a geometric distribution of pixels over intensity. Histogram equalization technique is considered a way to optimize the image by making gray output values uniformly distributed at all intensity levels. The features are observable and are invisible in the original image. This is done by the probability density function of gray level $r_{k}$ in an image and is illustrated in the following equations $[6,7]$ :

$$
\begin{gathered}
p_{k}\left(r_{k}\right)=\frac{n_{k}}{n} \\
s_{k}=T\left(r_{k}\right)=\sum_{j=0}^{k} p_{r}\left(r_{j}\right)
\end{gathered}
$$

where $p_{k}$ is the probability density function of gray level $r_{k}$ in an image, $\mathrm{n}_{\mathrm{k}}$ is the number of pixels having intensity $\mathrm{K}$, and the total number of pixels in image is $n$.

where $r_{k}$ show the gray levels of the input image and $s_{k}$ the gray levels of the processed image, $\mathrm{k}=0,1, \mathrm{~L}-1$.

Histogram Equalization method sometimes changes the original brightness of the image and can cause poor image quality. To get rid of these problems we list a number of ways below: Brightness preserving Bi-Histogram Equalization technique (BBHE) [8]. Dualistic Sub Image Histogram Equalization (DSIHE) [9]. The Minimum Mean Brightness Error Bi-Histogram Equalization (MMBEBHE) [10]. Recursive Mean Separate Histogram Equalization (RMSHE) [11].

\subsection{Gamma Correction}

Gamma correction techniques are a type of histogram modification (HM) technique that is flexibly obtained using a variable gamma correction $(\gamma)$ parameter. Gamma correction is a non-linear operation, which is used to correcting lightness or darkness of image [4].

\subsection{Histogram matching}

Histogram matching is utilized to get enhanced VS images. In general, VS images contain a limited distribution of histograms as opposed to visual images that have a better distribution of the histograms. The histogram matching method adjusts the histogram of the VS image to be redistributed across the above visual image range and adjusts the average and variance of the VS image based on its balance with the visual image for a better image[12-14].

\section{Proposed Approaches}

\section{B. This section presents the proposed approaches that enhance VS image.}

\subsection{The First Proposed Approach (CLAHE with Adaptive Gamma Correction)}

The first proposal is contrast limited adaptive histogram equalization with adaptive gamma correction. CLAHE is an improved technique of adaptive histogram equalization (AHE). Clipping limit is the value at which the histogram is cut and obtained from the following equation: [15]

$$
\beta=\frac{\mathrm{MN}}{\mathrm{L}}\left(1+\frac{\alpha}{100}\left(\mathrm{~S}_{\max }-1\right)\right)
$$

where $\beta$ is the clipping limit, $\mathrm{L}$ is the maximum intensity level of the original image, $S_{\max }$ is the maximum of the new distribution after the histogram equalization, $\alpha$ is the clipping factor, and $\mathrm{M}, \mathrm{N}$ are the dimensions of the VS image [16-18].

Adaptive gamma correction is used to improve the CLAHE output VS image. The improvement of image quality is not only used as an aesthetic form of its image. But often helps to improve image segmentation and identify features. While adjusting the contrast, the intensity value of each pixel in the original image is converted using a transfer function to form a better contrast image [19].

The adaptive gamma correction equation is derived by: [4]

$$
\mathrm{T}(i)=i_{\max }\left(i / i_{\max }\right)^{\gamma}
$$

where $i_{\max }$ is the maximum intensity of the input image. The intensity $i$ of each pixel in the input image is transformed as $\mathrm{T}(i)$ after performing equation (4).

The steps of the first proposal are listed as follows:

1. Divides the image into a number of continuous nonoverlapping sub-sections or blocks to reduce sub-sections artifacts between adjacent parts by interlocking interpolation or filtrating process.

2. Calculate the histogram for each section and implement the histogram equalization on each segment separately.

3. Perform a clip limit to avoid noise effect and computing cumulative distribution functions (CDF) for improved VS image. 
4. Apply adaptive gamma correction on the output of CLAHE image.

5. Estimate quality metrics after applying gamma correction, if quality metrics less than quality metrics of CLAHE repeat step 5 with change gamma correction.

6. If quality metrics bigger than quality metrics of CLAHE get VS image.

'Clip Limit' is a real scalar from 0 to 1 , the value $(\beta=0.1)$ from try and error is the best value to give optimum contrast for VS image.

By selecting optimal clip limit $(\beta=0.1)$ in CLAHE and adaptive gamma correction $(\gamma=1.3)$ illustrated in Fig. 1 and Fig. 4. It is clear that the first proposal achieves optimum contrast and best quality enhancement.

\subsection{The Second Proposed Approach (Homomorphic with Emphasis High Pass Filter)}

The Second Proposed Approach based on the second group transformation domain methods which uses the illumination and reflectance components for improving image contrast. We see the image $f\left(\mathrm{n}_{1}, \mathrm{n}_{2}\right)$ as the product of the light illumination incident and the reflectance of that object as shown below [20].

$$
f\left(n_{1}, n_{2}\right)=i\left(\left(n_{1}, n_{2}\right) r\left(n_{1}, n_{2}\right)\right)
$$

where $f\left(\mathrm{n}_{1}, \mathrm{n}_{2}\right)$ is the original image, $\mathrm{i}\left(\mathrm{n}_{1}, \mathrm{n}_{2}\right)$ is the light illumination incident on the object, $r\left(n_{1}, n_{2}\right)$ is the reflectance of that object. The first term in the above equation has small variations, but the second term has large variations, especially at intersections of unmatched parts. These features have the same Fourier transform properties so it can be used to image enhancement.

The basic concept is the separation between the illumination and reflectance components in logarithm domain realized in the form:

$$
\log \left(i f\left(n_{1}, n_{2}\right)\right)=\log \left(i\left(n_{1}, n_{2}\right)\right)+\log \left(r\left(n_{1}, n_{2}\right)\right)
$$

The enhancement image after homomorphic filtering as shown in eq. (7) below.

$$
f^{\prime}\left(n_{1}, n_{2}\right)=i^{\prime}\left(n_{1}, n_{2}\right) r^{\prime}\left(n_{1}, n_{2}\right)
$$

where $\mathrm{f}^{\prime} \quad\left(\mathrm{n}_{1}, \mathrm{n}_{2}\right)$ is the enhancement image, $\mathrm{i}^{\prime}\left(\mathrm{n}_{1}, \mathrm{n}_{2}\right), \mathrm{r}^{\prime}\left(\mathrm{n}_{1}, \mathrm{n}_{2}\right)$ show the light illumination and the reflectance of that enhanced object respectively.

The steps of the second proposal are summarized as follows:

1. Put the image in form illumination and reflectance components such as eq. 5 .

2. Apply log domain on the image to get the separation between the illumination and reflectance components.

3. Apply homomorphic with emphasis high pass filter on the image.
4. Changing and selecting the suitable values of the offset $(\partial=0.5)$ and scaling $(\chi=3)$ factors to adjust and improve quality of the image.

5. Restore the image to its normal shape by applying exponential on the image to get the enhanced image.

In homomorphic filtering applying the homomorphic with Gaussian high pass filter to the logarithmic domain VS image. It's an improved way of the Previous filter $\mathrm{H}(\mathrm{u}, \mathrm{v})$ that utilized as in the equation below [21].

$$
H \mathrm{e}(u, v)=\partial+\chi H(u, v)
$$

where $\mathrm{He}(\mathrm{u}, \mathrm{v})$ homomorphic with emphasis high pass filter, $\partial, \lambda$ offset and a scaling factor, and $\mathrm{H}(\mathrm{u}, \mathrm{v})$ homomorphic with Gaussian high pass filter. The resulting image is clearer and high contrast as shown in Fig. 4.

\subsection{The Third Proposed Approach (Homomorphic with Emphasis High Pass Filter and Histogram Equalization)}

The steps of the third proposal are listed as follows

1. Put the image in the form illumination and reflectance components and apply log domain on the image to get the separation between the illumination and reflectance components.

2. The homomorphic with emphasis high pass filter is executed for the videoscope image in logarithm domain to reinforcing reflectance and attenuating illumination.

3. Restore the image to its normal shape by applying exponential on the image to get the enhanced image.

4. Apply histogram equalization on the restored VS image, we get high quality and more details for the processed VS image as shown in Fig. 4. The simplest block diagram of the third proposed approach is summarized in the Fig.3

\section{Performance Evaluation Metrics}

The evaluation metrics which are used in the measurements are summarized as follows [22-24]:

\subsection{Entropy}

It is a statistical measure of randomness of the intensity of pixels in image. The larger the entropy, the more information the image carries. It is defined as: [22-24]:

$$
E=\sum_{i=0}^{255}-p_{i} \log _{2}\left(p_{i}\right)
$$

where $p_{i}$ is the probability density function of gray level in an image. The number of pixels having intensity ' $i$ ' is $n_{i}$, and the total number of pixels in image is $n, p_{i}=$ $\mathrm{n}_{\mathrm{i}} / \mathrm{n}$. 


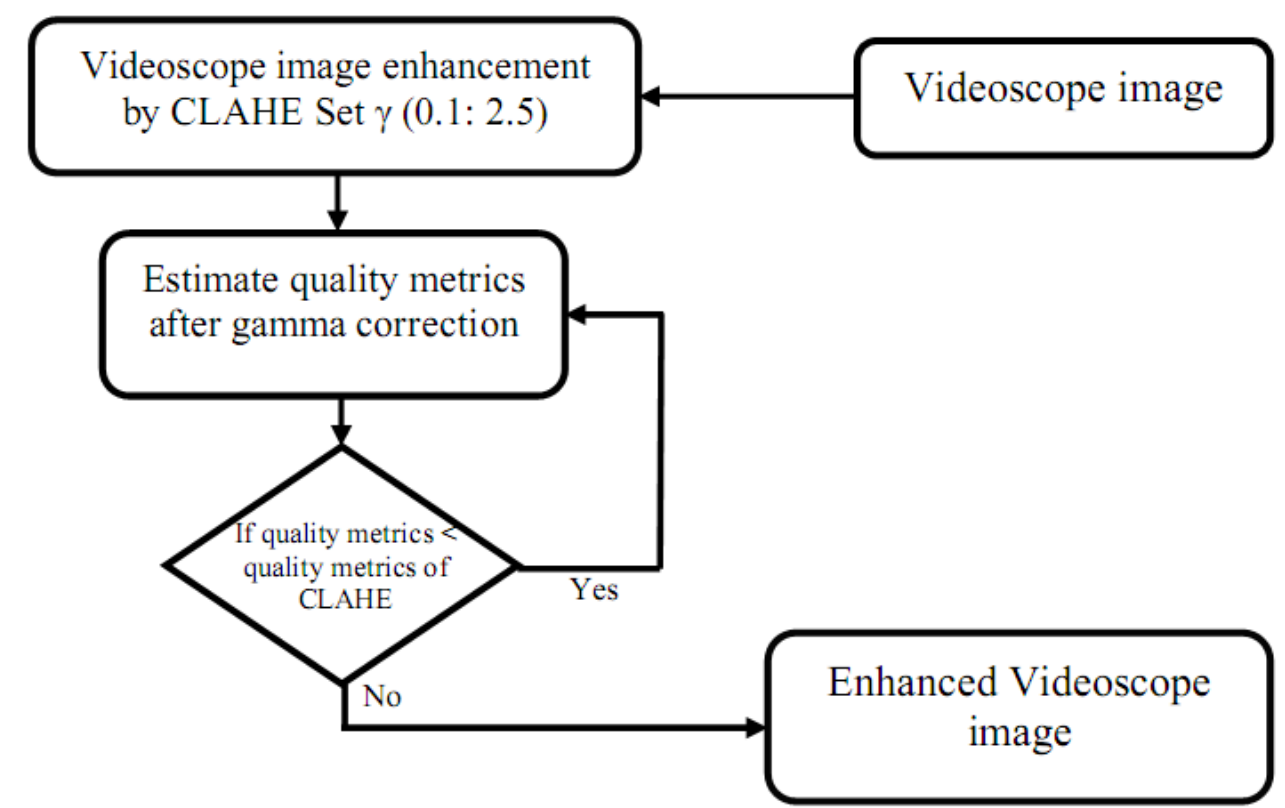

Fig. 1 Flow chart of VS image enhancement based on the first proposal

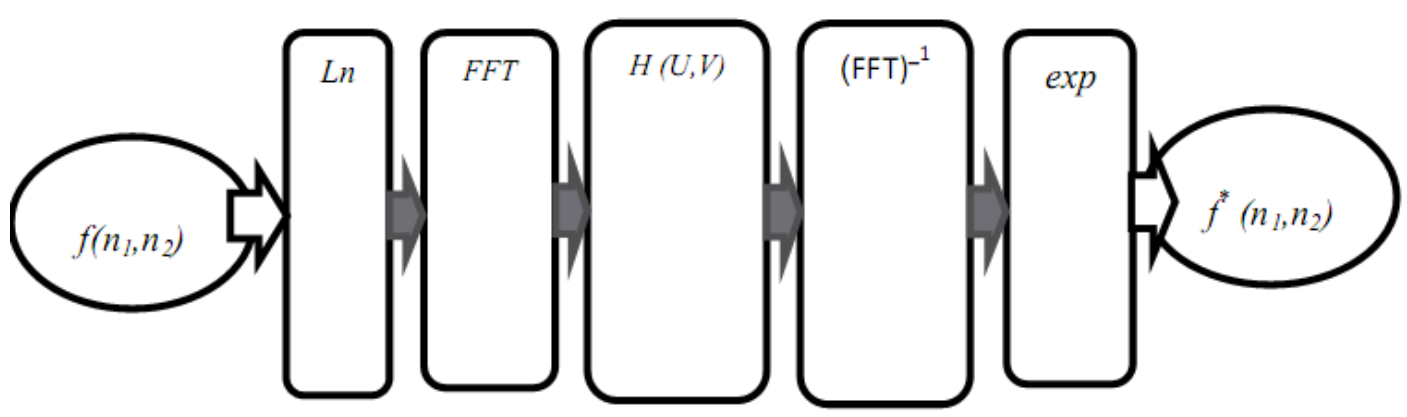

Fig. 2. General Homomorphic filtering approach for VS image

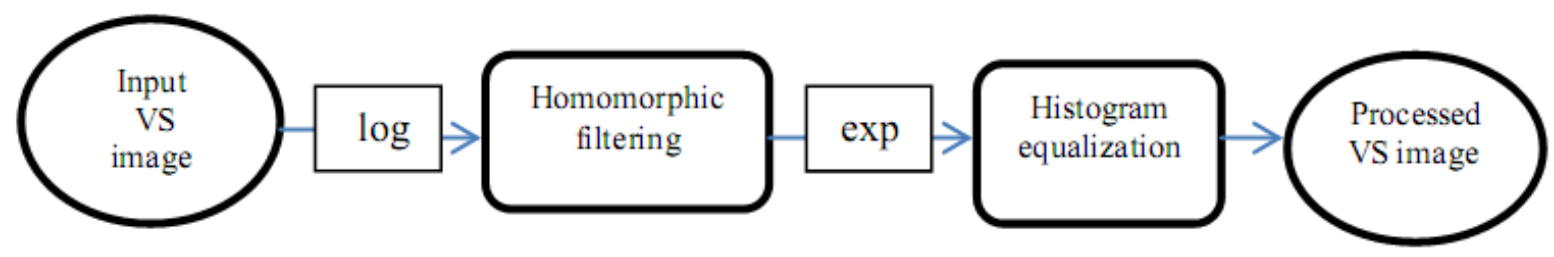

Fig. 3 the block diagram of the third proposed approach

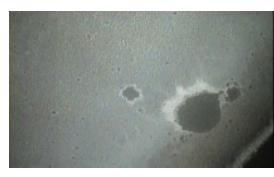

(a) original image

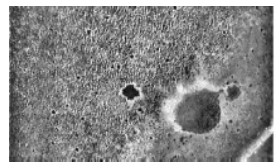

(d) CLAHE image

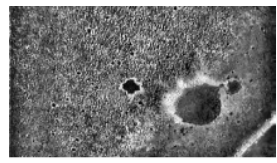

(g) first proposal

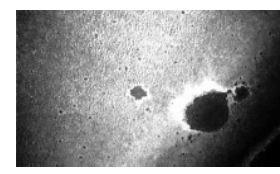

(b) hist eq image

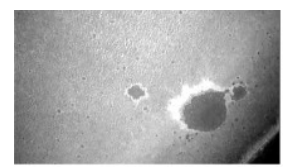

(c) hist match image

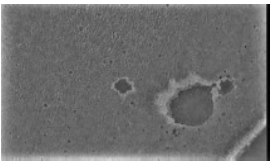

(e)gamma correction (f) Homomorphic GHPF
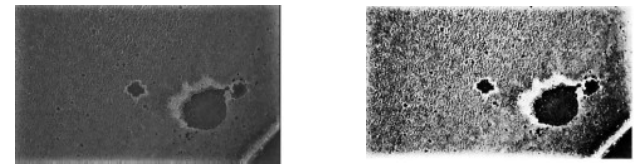

(h) second proposal

(i) third proposal

Fig. 4 Results of the VS image experiment 
Table 1 Numerical results of the VS image experiment

\begin{tabular}{|c|c|c|c|c|}
\hline Quality metrics & Entropy & $\begin{array}{l}\text { Average } \\
\text { gradient }\end{array}$ & $\begin{array}{l}\text { Contrast } \\
\text { improvemen } \\
\mathrm{t} \text { factor }\end{array}$ & $\begin{array}{l}\text { Sobel edge } \\
\text { magnitude }\end{array}$ \\
\hline Original image $\quad$ size $(570 \times 968)$ & 6.8867 & 0.0100 & 0.1487 & 0.1071 \\
\hline Histogram equalization image & 5.9836 & 0.0270 & 0.3377 & 0.2872 \\
\hline Histogram matching image & 6.8197 & 0.0142 & 0.1520 & 0.1820 \\
\hline CLAHE image & 7.6082 & 0.0534 & 0.6204 & 0.5694 \\
\hline $\begin{array}{l}\text { The first Proposal, gamma set point } \\
=1.3\end{array}$ & 7.6367 & 0.0557 & 0.7220 & 0.5946 \\
\hline Adaptive gamma correction image & 6.7998 & 0.0151 & 0.1673 & 0.1615 \\
\hline $\begin{array}{l}\text { Homomorphic with Gaussian high } \\
\text { pass filter }\end{array}$ & 3.9089 & 0.0071 & 2.4113 & 0.0757 \\
\hline The second Proposal image & 7.1003 & 0.0316 & 0.7180 & 0.3377 \\
\hline The third proposal & 5.3965 & 0.0642 & 0.7223 & 0.6867 \\
\hline
\end{tabular}

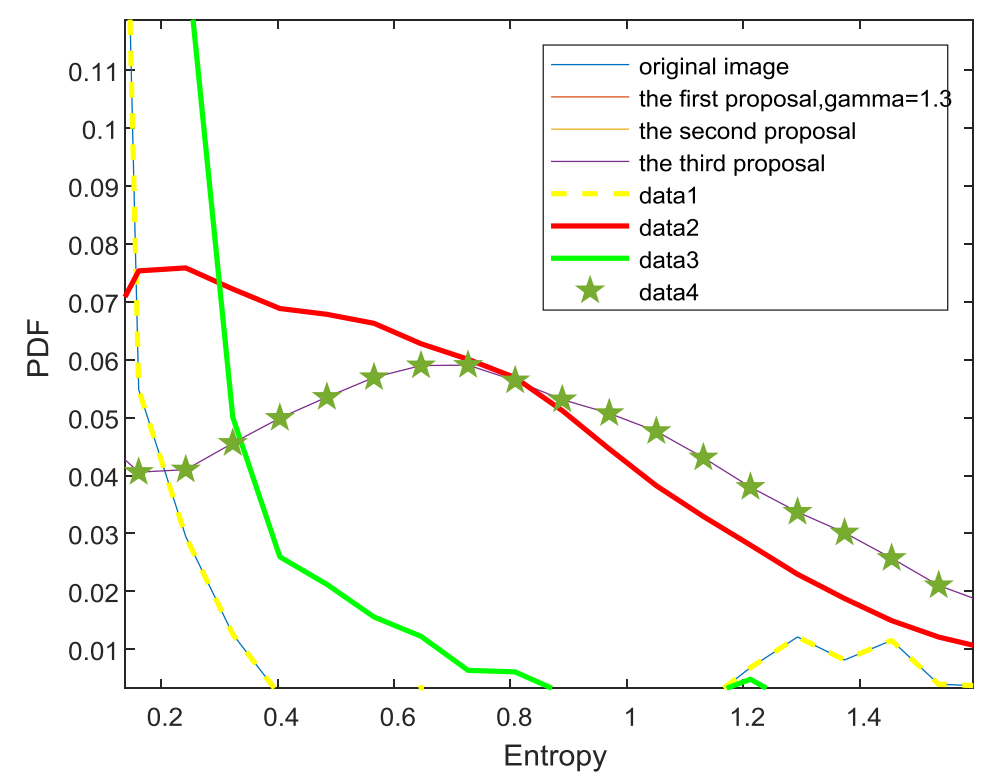

Fig. 5 Results of spectral entropy of the VS image experiment

\subsection{The contrast enhancement factor}

By increasing contrast, image quality increases. It is defined as [22-24]:

$$
c_{I F}=\frac{\left|c_{o}-c_{e}\right|}{c_{o}}
$$

where $c_{\mathrm{o}}$ is input image contrast, and $\mathrm{c}_{\mathrm{e}}$ is the processed VS image contrast.

\subsection{Average Gradient}

It is the factor that represents the detail contrast and structure variation. When it increases, more accurate VS image. It is defined as [22-24]:

$A G=\frac{1}{M N} \sum_{m=1}^{M} \sum_{n=1}^{N} \sqrt{\frac{\left.\left(\frac{\partial f}{\partial x}\right)^{2}+\left(\frac{\partial f}{\partial y}\right)^{2}\right)}{2}}$

where $\mathrm{M}$ and $\mathrm{N}$ are the dimensions of the image. 
4.4 The Sobel Edge Magnitude

It is used for edge detection of the VS image. It is defined as [22-24]:

$$
\Delta f=\sqrt{f_{x}^{2}+f_{y}^{2}}
$$

where $f_{x}$ and $f_{y}$ are the horizontal and vertical derivative approximations VS images, respectively.

\subsection{Numerical and Simulation Results and Discussion}

\subsubsection{Numerical Results:}

We utilize one experiment on video scope image with size (570x968) to calculate the numerical and simulation results of three proposals. The results of three proposed approach are shown in Fig. 4 present the original VS image with size (570x968), the VS image histogram equalization, histogram matching image, the VS image after CLAHE, the enhanced VS image using the first proposed algorithm with gamma $=1.3$, adaptive gamma correction image, homomorphic with Gaussian high pass filter, homomorphic with emphasis high pass filter (the second proposed approach), and the third proposed approach. The numerical results are listed in Table 1 and the simulation results of spectral entropy in Fig. 5.

By selecting optimal clip limit $\beta(0.1)$ in CLAHE and adaptive gamma correction $\gamma$ (1.3), the results obtained reveal the ability of first proposal to improvement of the contrast improvement factor and it succeeded in implementing best details and provides a better visual quality of the output processed VS image. By selecting the suitable values of the offset $(\partial=0.5)$ and the scaling $(\chi=$ 3 ) factors for the second proposal. It is clear that the second proposed approach succeeded in giving best contrast improvement and keeping information unchanged of input VS image. By utilizing histogram equalization on homomorphic with emphasis high pass filter VS image (the third proposed approach) provides more details and more accurate and provides a better visual quality of the output VS image.

From the numerical results listed in Table 1 it is clear that the first proposal achieves better entropy than the second and third proposals, while the third proposal achieves better edge magnitude and Average gradient than the first and second proposals, but suffers from the smallness of the entropy. The enhanced videoscope images using the three proposals of these utilized experiment achieved both qualitative and quantitative estimation.

\subsubsection{Simulation Results}

where The spectral entropy describes the complexity of a system, PDF is the Probability density function. It's clear that the distribution of probability density functions of the spectral entropy of the three proposed approaches for all utilizing experiments shown above figures better than original image.

And finally it's clear that from executed experiment the simulation results as shown in figure (5) the first proposal achieves the best distribution of probability density functions of the spectral entropy followed by the third proposal while the second proposal is the lowest.

\section{Conclusion}

This paper presented three efficient proposed approaches for contrast and quality enhancement of videoscope images. The first proposal is based on contrast limited adaptive histogram equalization with adaptive gamma correction. The CLAHE adjusts the differences in the dynamic range of sub-images, and the adaptive gamma correction achieves optimum contrast enhancement and more details for the VS images. The second proposal is homomorphic with emphasis high pass filter of VS images through logarithmic transform using this filter with different offset and a scaling factor. It attenuates the illumination, while the reflectance is magnified. The obtained results showed more details and better contrast improvement. The third proposal is homomorphic with emphasis high pass filter and histogram equalization. We applied histogram equalization on the second proposal for VS images. The obtained results showed more details and more accurate VS images. The three proposals can be suggested for the industrial videoscope devices.

\section{References}

[1] KARL STORZ, "Manual videoscope", GmbH \& Co. KG, 2009.

[2] h. Lidong, Z. Wei, W. Jun and S Zebin, "Combination of contrast limited adaptive histogram equalization and discrete wavelet transform for image enhancement", 2015. [3] R. Gonzalez and R. Woods, "Digital Image Processing", Vol. 3. London: Pearson Education, 2009.

[4] C. Shih, C. Fan and S. Yi, "Efficient Contrast Enhancement Using Adaptive Gamma Correction with Weighting Distribution", 2013.

[5] H. I. Ashiba, H. M. Mansour,H. M. Ahmed, M. I. Dessouky, M. F. El-

Kordy, O. Zahran \& Fathi E. Abd El-Samie, "Enhancement of IR images using histogram processing and the Undecimated additive wavelet transform", Multimedia Tools and Applications, doi.org/10.1007/s11042-018-6545-9, 2018.

[6] R. Greg, B. Mitta and S. Grag, "Histogram equalization techniques for image enhancement", International Journal of Electronics \& Communication Technology, 2(1), 107-111, 2011.

[7] A. Singh, "Contrast Enhancement for Cephalometric Images using Wavelet-based Modified Adaptive Histogram Equalization", Soft Computing Journal, doi.org/doi:10.1016/j.asoc.2016.11.046,2016,2016.

[8] K.Akilaa, L.S.Jayashreeb, A.Vasukic," Mammographic image enhancement using indirect contrast enhancement techniques - A comparative study ",Elsevier Procedia Computer Science, 2015.

[9] S. Sophoan," Color Image Enhancement by Using Dynamic Piecewise Linear Transformation", sophoan.ssse2018@tggs.kmutnb.ac.th,2018.

[10] S.-D. Chen and A.- R. Ramli, "Minimum mean brightness error bi-histogram equalization in contrast 
enhancement", IEEE Transactions on Consumer Electronics, November, 2003.

[11] S.-D. Chen and A.-R. Ramli, "Contrast enhancement using recursive mean-separate histogram equalization for scalable brightness preservation", IEEE Transactions on Consumer Electronics, 2003.

[12] Y. Hel, H. Hel and E. David, "Fast template matching in non-linear tone-mapped images", In Computer vision (ICCV), international conference on IEEE (pp. 1355-1362), 2011.

[13] J.-C Russ "The image processing handbook", (5rd edn.). CRC Press, 2007.

[14] J.-P Rolland, V. Vo, B. Bloss and C.-K Abbey. "Fast algorithms for histogram matching: Application to texture synthesis", Journal of Electronic Imaging, 2000.

[15] H.-I Ashiba, H.-M Mansour, H.-M Ahmed, M.-F ElKordy, M.-I Dessouky and F.-E.-A. El-Samie, "Enhancement of infrared images based on efficient histogram processing", Multimedia Tools and Applications, https://doi.org/10.1007/s11277-017-4958-9, 2018.

[16] S.-K Shame and S.-R.-K Vadali, "Enhancement of Di-abetic retinopathy imagery using contrast limited adaptive histogram equalization", International Journal of Computer Science and Information Technologies, 2(6), 2694-2699, 2011.

[17] J.A Stark, "Adaptive image contrast enhancement using generalizations of histogram equalization", IEEE Transactions on Image Processing, 9(5), 889-894.doi: https://doi.org/10.1109/83.841534, 2000.

[18] W. Zhiming and T.- A Jianhua, "Fast implementation of adaptive histogram equalization", In IEEE, ICSP proceedings, 2006.

[19] R. Gonzalez and R. Woods, "Digital Image Processing", 2nd edition, Upper Saddle River, 2001.

[20] R. Gonzalez, R. Woods and S. Eddins, "Digital Image Processing Using MATLAB", 2004.

[21] R. Gonzalez and R. Woods and S. Eddins, "Digital image processing using MATLAB", 2009.

[22] T. T. Neo, "Fusion of Night Vision and Thermal Images', M.Sc. Thesis, Naval Postgraduate School, Universityof New South Wales, Australia, 2006

[23] S. Yin, L. Cao, Q. Tan, and G. Jin, "Infrared and Visibl Image Fusion based on NSCT and Fuzzy Logic ", in Proc. of the IEEE Int. Conf. on Mechatronics and Automation, pp. 671-675, August 4-7, 2010.

[24] J. Zhuqing, "Study of Multi-Source Image Fusion Method in Transform Domain", 2011. 